Astronomy Letters, vol. 00, XX-XX, 2015

\title{
FRACTAL PROPERTIES OF STELLAR SYSTEMS AND RANDOM FORCES
}

\author{
O. Chumak ${ }^{1}$ and A. Rastorguev ${ }^{1,2}$ \\ 1 Sternberg State Astronomical Institute, M. V. Lomonosov Moscow State \\ University, Universitetskiy Prospekt 13, Moscow 119992, Russia; \\ chumako@sai.msu.ru,rastor@sai.msu.ru \\ 2 Physics Faculty, M. V. Lomonosov Moscow State University, \\ 1 Bald. 2, Leninskie Gory, 119991 Moscow, Russia; rastor@sai.msu.ru
}

Received: 2015 October 26

\begin{abstract}
The nearest neighbor distribution Chandrasekhar (1943) is generalized to fractal stellar systems.For such systems an asymptotic distribution of the magnitude of large random forces and a formula for the effective mean interparticle spacing are derived. It is shown that in the case of a power-law distribution of conditional density the derived asymptotic fully agrees with the results obtained in terms of a general approach. It is concluded that large random forces in a fractal stellar medium are due entirely to the nearest neighbors (clumps) located inside the sphere of the effective radius determined from the generalized Holtsmark distribution.
\end{abstract}

Key words: fractal nature of the distribution of stars, random forces, nearest neighbor distribution, random force distribution, relaxation

\section{INTRODUCTION}

In this paper we investigate random forces acting on a test star that are caused by multiscale stellar density fluctuations (fractal stellar substructures). Currently, the fractal structure f star-forming regions on spatial scales from 1 to 200-300 pc is well established by observations of the young population in the Milky Way and other galaxies (Efremov \& Elmegreen, 1998; Elias et al., 2009; Sanchez et al., 2010; Elmegreen et al., 2014; Gouliermis et al., 2014). Our earlier analysis of the Geneva-Copenhagen survey of FG-type dwarfs (Nordstrem et al., 2004; Holmberg et al., 2007, 2009) revealed similar fluctuations with magnitudes substantially exceeding those expected for uniform random (Poisson) space distributions to be present in the solar neighborhood, i.e., in the Galactic field. The fractal nature of the distribution of FG-type dwarfs is believed to also reflect the features of the overall distribution of local stars: we showed (Chumak \& Rastorguev, 2015) that the space distribution of FG-type dwarfs in the solar neighborhood differs significantly from uniform random distribution on all available scale lengths (from 1 to $20 \mathrm{pc}$ ), i.e., that the stellar medium demonstrates significant and well-defined "coarse-grained structure", which can be described by the average Hausdorff dimension of $D \approx 1.23$. One of the main problems in the study of the kinetics of a system of gravitating point masses is the analysis of the forces that act on test 
particles. In stellar dynamics such an analysis is traditionally performed by investigating the statistical and dynamic properties of the gravity force produced by the medium surrounding the test star. The results of such an analysis allow one to derive stochastic and kinetic equations of stellar dynamics in different approximations. The stellar dynamics eq uations derived in terms of such an approach may have different forms depending on the formulation of the problem and assessment of the effect of various factors. The fundamental nature of the problem of forces acting on a test star becomes clear from the fact that the corresponding equations form the basis for all the main results in stellar dynamics including the relaxation theory. Analysis of random forces usually begins with selecting a model for the medium surrounding the test particle. The simplest model is that of infinite uniform static medium that is (1) isotropic on large scales, (2) with limitedfluctuations, (3) consisting of point particles of various masses randomly distributed according to Poisson's law, and (4) without mutual correlations. Such a model is known Chandrasekhar (1943) to imply the Holt smark distribution of the magnitude of the random force acting on a test star. Based on the Holtsmark distribution one can derive not only the asymptotic formulas for the distribution of force, but also compute the most probable random force for the model of the medium considered. However, the second moment of the distribution (dispersion) diverges, makings it difficult to construct consistent kinetics of the stellar medium in terms of such a model. The allowance for spatial correlations may in some cases result even in the divergence of the first moment Gabrielli et al.(1999). As is well known, the Fokker-Plank approximation also results in divergences in the formulas for the force of dynamic friction and components of the diffusion tensor, which can be prevented by artificially constraining t he Coulomb logarithm by imposing certain distance limits (the size of the system or the average spacing between the stars). The classic model mentioned above yields practically infinite relaxation times exceeding the age of the system by several orders of magnitude. These relaxation times are inconsistent with the results of observations, which imply the well-mixed state of the stellar population. Ogorodnikov (1958) called the problem of unrealistically large relaxation times the fundamental paradox of stellar dynamics. Thus this model, which yielded excellent results for quasi-neutral uniform plasma, proved to be of little use in the case of gravitating stellar medium where it can be considered only as a first approximation despite the known similarity of the two types of media. All this motivates the researchers to construct model distributions of gravitating masses that would more adequately describe the observed distribution. Such models are believed to eventually eliminate the divergences and enable the development of internally consistent kinetic theory of stellar medium that would agree better with observational data. The class of anisotropic models fits into this category. It is well known from observations that stellar medium in stellar systems is almost always anisotropic because of the presence of external field. Thus Chumak \& Rastorguev (2014) showed that with the anisotropy of velocity distribution taken into account the classic formula for relaxation can be generalized resulting in strong reduction of the relaxation time compared to standard theory. The second important factor that determines the development of kinetic processes in stellar medium is its structural inhomogeneity. Multiscaled correlation structures in gravitating media have always been a particularly popular topic among the researchers. The origin of these structures, their physical and dynamic properties have been studied in such depth and detail as permitted by the available observing techniques, physical concepts, and the state of the devel- 
opment of the methods of mathematical analysis. It is evident that young stellar groupings - open clusters and associations - may still contain residual structures that formed during the gravitational fragmentation of protoclusters from molecular clouds. These residual structures include binary and multiple systems, many of which will disrupt as a result of stellar encounters. However, because of the very nature of gravitational interactions between the stars, gravitationally unbound short-lived substructures may form in an initially quasi-uniform medium. The discovery of such structures in the solar neighborhood (Chumak \& Rastorguev, 2015 ), i.e., among the rather old population of the Galactic disk, is indicative of the efficiency of this mechanism, which appears to be universal. If this is the case, estimating the effect of fractality on the kinetics of stellar systems, i.e., its contribution to the distribution of random force, should be a relevant and interesting task.

\section{STOCHASTIC HIERARCHIES IN GRAVITATING MEDIA}

There is nothing unusual in the existence of fractal structures in the stellar medium. Carpenter (1938) found the number of galaxies in a cluster, $N(r)$, to depend on $r$ as $\sim r^{1.5}$ rather than $r^{3}$, as would be expected for uniform space distribution of galaxies. In other words, the number density of galaxies varies as $n(r) \sim N(r) / r^{3} \sim r^{-1.5}$, i.e., it decreases with increasing characteristic size in accordance with a fractional-power law with exponent $(-3 / 2)$. Carpenter concluded that clusters of galaxies are not quasi-isolated objects, but rather sort of concentrations in some non-uniform but non-random limited-density distribution. De Vaucouleurs (1970) repeated Carpenter's analysis using more up-to-date data (de Vaucouleurs et al., 1991) and concluded that the distribution of galaxies, on the average, obeys the law $n(r) \sim r^{-1.7}$; he generalized Carpenter's conclusions extending his results not only to clusters of galaxies, but also to the entire galaxy medium. After de Vaucouleurs (1970) paper and many other studies published since then by different authors the fractal structure of the world of galaxies became the generally accepted model in extragalactic astronomy (see, e.g., Wu et al., 1999). It turned out that the world of galaxies is organized hierarchically, and therefore any observer associated with an object that is a part of this hierarchy sees the average density around him to decrease with distance. At the same time, there is no special location in this medium, i.e., any sufficiently large and equal volumes have the same average density irrespective of their relative location. This density can be referred to as invariant conditional density (CD). In the case of synchronous variation the size of these $\mathrm{CD}$ volumes varies with characteristic size $r$ as

$$
n(r) \sim r^{-\alpha}
$$

where $\alpha \approx 1.7$. This is what is called the Carpenter-de Vaucouleurs law of galaxy clustering. Based on the results of de Vaucouleurs, Mandelbrot $(1977,1988)$ interpreted the law represented by eq. (1) as a special case of stochastic self-similarity of three-dimensional fractal sets, which obeys relation:

$$
N(r) \sim r^{3-\alpha}=r^{D}
$$

. Here $D$ is referred to as the fractal (Hausdorff) dimension of the medium. Mandelbrot showed that depending on the properties of the medium such fractal structures can occur in different gravitating media and may have fractal dimensions in 
the $0 \leq D \leq 3$ interval, i.e., exponent $\alpha$ may vary in the interval from -3 to 0 . Numerous numerical simulations of the dynamic evolution of self-gravitating systems performed using Nbody algorithms also confirmed the formation of stochastic hierarchical structures in initially uniform self-gravitating systems. These hierarchical structures are similar to those actually observed both in the galaxy and interstellar media over a range of spatial scales spanning several orders of magnitude (see, e.g., Semelin aand Combes, 2000, 2002; Bottaccio et al., 2003, and others). In our earlier study (Chumak and Rastorguev, 2015) based on an analysis of the space distribution of stars in the Geneva-Copenhagen survey, we found that the solar neighborhood in the Galaxy has a fractal structure with a mean Hausdorff dimension of $D \approx 1.23$. We also estimated the dependence of conditional density on distance from each star of the sample, namely, we determined the number $N(r, i)$ of stars inside spheres of increasing radii $r$ centered on i-th star of the sample, and then computed the corresponding conditional density $n(r, i)$. It is evident that for each radius $r$ there are some maximum, mean, and minimum values of conditional density. For the solar neighborhood these three series of $n(r)$ values can be with high degree of confidence fitted by power laws:

$$
n(r)=h r^{-\alpha}
$$

The parameters of dependence $(3)$ are equal to $h \approx 0.036$ and $\alpha \approx 0.948$ (at a Pearson confidence level of $R^{2} \approx 0.867$ ) for the series of maximum conditional density values $n_{\max }(r) ; h \approx 1.644$ and $\alpha \approx 1.769\left(R^{2} \approx 0.991\right)$ for the mean conditional density $n_{\text {mean }}(r)$, and $h \approx 0.483$ and $\alpha \approx 3.007\left(R^{2} \approx 0.9998\right)$ for the minimum conditional density $n_{\min }(r)$.

The existence of minimum conditional density values indicates that in the stellar medium there always regions where the mass inside a sphere surrounding a star is practically independent of the radius of this sphere $(\alpha \approx 3.0)$. In other words, voids occur on all the scales considered (from 1 to $20 \mathrm{pc}$ ), indicating the presence of intermittency in the stellar medium.

The average Hausdorff dimension $(D=3-\alpha)$ of the space distribution of the FG-type dwarfs studied is equal to $D \approx 1.23$. Figure 1 shows the log-log plot of the distance dependence of conditional density averaged over all stars of the sample and corresponding to this Hausdorff dimension.

Given the lack of similar data for the space distribution of other stars in the local volume we assess the effect of fractality on stellar kinetics assuming that the fractal properties of the local sample of FG-type dwarfs are representative of the entire stellar population of the Galactic disk.

\section{ASYMPTOTIC LAW OF THE DISTRIBUTION OF THE MAGNITUDE OF RANDOM FORCE IN A FRACTAL MEDIUM IN THE NEAREST-NEIGHBOR APPROXIMATION}

As we pointed out above, Chandrasekhar (1943) derived an exact solution for the distribution of the magnitude of fluctuating random force in the case of uncorrelated (Poissonian) static random space distribution of stars. This solution for the magnitude of the force has the form of the Holtsmark distribution:

$$
W(|\vec{F}|)=H(\beta) a^{3 / 2}
$$




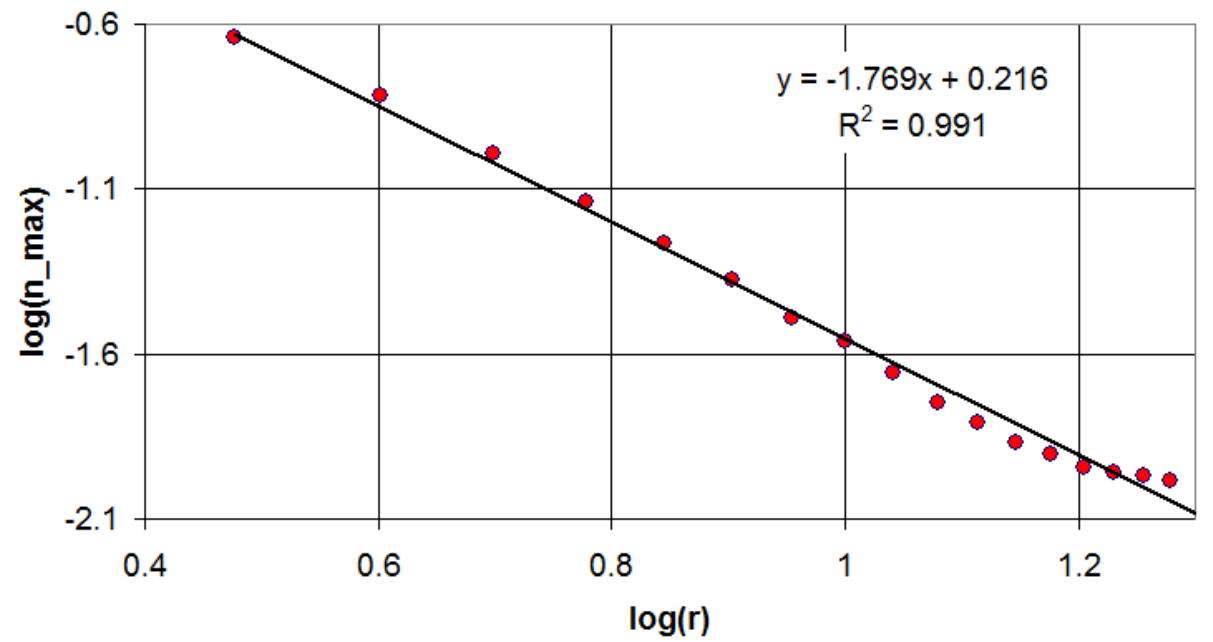

Fig. 1. Average dependence of the logarithm of conditional density on the logarithm of the radius of the volume based on the data of the Geneva-Copenhagen survey

where

$$
H(\beta)=\frac{2}{\pi \beta} \int_{0}^{\infty} \exp \left[-(x / \beta)^{3 / 2}\right] x \sin (x) d x
$$

and in the case of equal-mass stars

$$
a=(4 / 15)(2 \pi G m)^{3 / 2} n
$$

The dimensionless force is equal to

$$
\beta=|\vec{F}| / a^{2 / 3}
$$

Chandrasekhar (1947) showed that the asymptotics of the Holtsmark distribution in the approximation of large random forces exactly coincides with the distribution of the random force acting on a unit-mass test star and due to the nearest neighbor of mass $m$ located at distance $r$ :

$$
|\vec{F}|=\frac{G m}{r^{2}}
$$

The asymptotic formula for $W(|\vec{F}|)$ can be derived from the nearest neighbor distance distribution $w(r)$ :

$$
\begin{gathered}
w(r) d r=4 \pi n \exp \left(-4 \pi r^{3} n / 3\right) r^{2} d r \\
W(|\vec{F}|) d|\vec{F}|=4 \pi n(G m)^{3 / 2} \exp \left[4 / 3 \pi n(G m)^{3 / 2}|\vec{F}|^{-3 / 2}\right]|\vec{F}|^{-5 / 2} d|\vec{F}|
\end{gathered}
$$

We now generalize the Holtsmark law to the case of fractal distribution assuming that the force acting onto the test star is also determined by its nearest neighbor exclusively and taking into account the clumpy structure of the stellar medium. 
To this end, the distribution of the nearest-neighbor distance should be derived using the distance dependence of average density in the form of formula (3):

$$
w(r)=\left[1-\int_{0}^{r} w(r) d r\right] 4 \pi r^{2} n(r)=4 \pi h\left[1-\int_{0}^{r} w(r) d r\right] r^{D-1}
$$

implying that:

$$
\frac{d}{d r}\left[\frac{w(r)}{4 \pi h r^{D-1}}\right]=-4 \pi h r^{D-1} \frac{w(r)}{4 \pi h r^{D-1}}
$$

According to formula (11),w(r) $\rightarrow 4 \pi h r^{D-1}$ at $r \rightarrow 0$, and it therefore follows from equation (12) that

$$
w(r) d r=4 \pi h \exp \left(-\frac{4 \pi h}{3} r^{D}\right) r^{D-1} d r
$$

This is the analog of formula (10), i.e., the generalization of the nearest-neighbor distribution for the case of fractal mass distribution. Further, given formula (8), we derive from equation (13), after simple rearrangement, the following generalization of formula (10) for the case of power-law density distribution (3):

$$
W(|\vec{F}| \mid D) d|\vec{F}|=4 \pi h(G m)^{D / 2} \exp \left[-\frac{4 \pi h}{3}\left(\frac{G m}{|\vec{F}|}\right)^{D / 2}\right]|\vec{F}|^{-\frac{D+2}{2}} d|\vec{F}|
$$

where, as above, $D$ is the fractal dimension.

In the limit of uniform medium $(\alpha \rightarrow 0, D \rightarrow 3, h \rightarrow n)$ formula (14) goes over into the standard Holtsmark distribution (10).

In the limit of strong fields $(|\vec{F}| \rightarrow \infty)$ we obtain from formula (14) the following formula for the magnitude of random force $F=|\vec{F}|$ :

$$
W(F \mid D) \approx 4 \pi h(G m)^{D / 2} F^{-\frac{D+2}{2}}
$$

We now denote the dimensionless argument of the exponential function in formula (14) as $x=\frac{4 \pi h}{3}\left(\frac{G m}{F}\right)^{D / 2}$ to rewrite formula (14) in the form

$$
W(F \mid D) F=3 x e^{-x}
$$

or

$$
A W(F \mid D)=(3 x)^{(D+2) / D} e^{-x}
$$

where

$$
A=\frac{(4 \pi h)^{2 / D}}{G m}
$$

Figure 2 shows the standard Holtsmark law (the lower curve) and our derived distribution (the upper curve) that takes into account the fractal nature of the distribution of local stars with the Hausdorff dimension $D \approx 1.23$ (the upper curve).

It is evident from formulas (16) that probability distribution $W(F)$ decreases most rapidly with force magnitude in the case of uniform distribution $(D=3)$. 


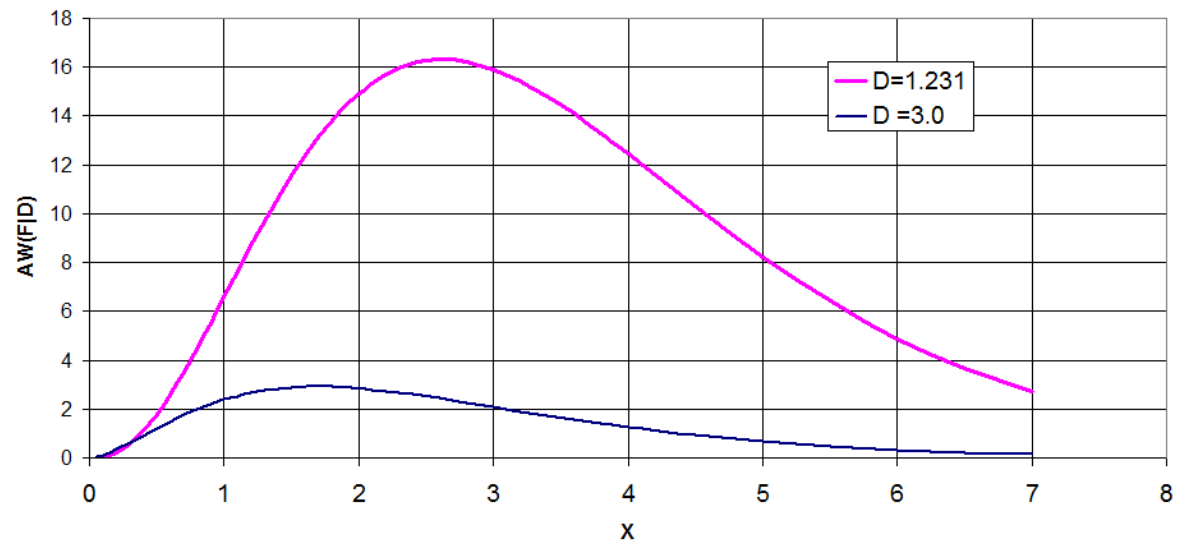

Fig. 2. Average dependence of the logarithm of conditional density on the logarithm of the radius of the volume based on the data of the Geneva-Copenhagen survey

In this sense the Holtsmark random distribution is the lower limit for $W(F)$. Any real distributions of gravitating masses with the mean conditional density of the form of equation (3) with $\alpha>0(D<3)$ should generate more slowly decreasing random force distributions $W(F)$, thereby demonstrating that strong fields play more important role in the kinetics of gravitating medium. Furthermore, it is evident from Fig. 2 that the most probable normalized random force is substantially (by a factor of about 1.7) greater than the value computed for the standard Holtsmark distribution.

The distribution of random force acting on a test star in the solar region can be computed in the nearest neighbor approximation by substituting into formula (14) the Hausdorff dimension $D \approx 1.23$ determined above. To apply distribution law (14) to other parts of the Galaxy and to open and globular clusters, we should ensure that power law (3) holds for these regions. Needless to say that fractal dimension $D$ differs in each particular case.

\section{ESTIMATING THE EFFECTIVE INTERPARTICLE SPACING FOR THE FRAC-}

TAL MEDIUM

The effective interparticle spacing $\mathrm{rm}$ and characteristic effective time tm of mutual pairwise encounters in a fractal gravitating medium can be determined from formula (13) for the distribution of the nearest neighbor distance. By definition

$$
r_{m}=\int_{0}^{\infty} r w(r) d r
$$

where probability $w(r)$ should be computed by formula (13). Note that for convenience of further computations $w(r) d r$ is better to rewrite in the form

$$
w(x) d x=\frac{3}{D} e^{-x} d x
$$

where $x=\frac{4 \pi h}{3} r^{D}, D=(3-\alpha)$ is the Hausdorff fractal dimension, and $h$ is the density parameter in formula (3). Given formula (18), we then derive from formula 
(17) the following exact estimate for $r_{m}$

$$
r_{m}=\frac{3}{D}\left(\frac{4 \pi h}{3}\right)^{-1 / D} \int_{0}^{\infty} e^{-x} x^{1 / D} d x=\frac{3}{D}\left(\frac{3}{4 \pi h}\right)^{1 / D} \Gamma\left(\frac{D+1}{D}\right) .
$$

In the limit $D \approx 3, h \approx n$ - (uniform random Poisson medium) we obtain the well-known Chandrasekhar's (1943) estimate for the mean interparticle spacing

$$
r_{m}=\left(\frac{3}{4 \pi n}\right)^{1 / 3} \Gamma\left(\frac{4}{3}\right) \approx 0.554 n^{-1 / 3} .
$$

Substituting our parameter estimates for the space distribution of the nearsolar sample of FG-type dwarfs, $h \approx 1.644$ and $D \approx 1.23$, into formula (19) yields an effective interparticle spacing of $r_{m} \approx 0.48 p c$ for the fractal random stellar medium. Let us compare it with traditional estimates of the mean distance between stars. Published estimates of local stellar density differ by about a factor of two. Thus the well-known handbook by Allen and $\operatorname{Cox}(2004)$ gives $n \approx 0.141 p c^{-3}$. Bahcall and Soneira (1986) normalize their Galaxy model to the local space number density of $n\left(R_{0}\right) \approx 0.13 p c^{-3}$. Yanny and Gardner (2013) estimate the local stellar number density to be $n \approx 0.1090 .002 p c^{-3}$. When building their model of the Galaxy, Roben et al. (2003) adopt the local mass density of $\rho \approx 0.076 \mathrm{M}_{0} p c^{-3}$, which, if converted into stellar number density, also agrees with the above estimates. Substituting the extreme values 0.109 and $0.141 p c^{-3}$ into classical formula (20) yields an average distance between the stars of $r_{m} \approx 1.16$ and $1.06 p c$, respectively, which is almost twice greater than our preliminary estimate obtained with the allowance for the fractal structure of the distribution of FG-type dwarfs.

We estimate the contribution of FG-type dwarfs to the total local stellar density to be $0.03-0.04 p c^{-3}$, i.e., from 40 to $25 \%$. If we assume that the fractal space distribution of FG-type dwarfs is representative of that of all stars in the solar neighborhood then to more accurately estimate the effect of fractality in the full sample of disk stars the parameter $\mathrm{h}$ in formula (19) for the effective distance between the stars should be increased proportionally by a factor of 2.5 - 4 compared to our sampled value of $h \approx 1.644$, i.e., up to $h \approx 4-7$. In this case more realistic estimates of the effective interparticle spacing $r_{m}$ for the entire local star sample decrease to 0.27 and $0.15 \mathrm{pc}$, respectively, which is about a factor of four to six less than the observed mutual distances between stars in the nearest solar neighborhood. This result is due to the fact that fractal model takes into account the gravitational clustering ("clumpiness") of the stellar medium in regions with the radii of several tens of pc.

The characteristic time scale of the fluctuations of irregular forces that are due to stellar encounters is on the order of $t_{m} \approx r_{m} / v_{m}$, where $v_{m}$ is the mean relative velocity of particles. Given that for a fractal medium $r_{m}$ can be almost by one order of magnitude smaller than the corresponding value for a uniform Poisson medium, it may be assumed that in fractal media diffusion processes in the velocity space should play more important part than classical diffusion and dynamic friction. This problem requires special analysis.

\section{DISCUSSION}

Numerical simulations of the evolution of a medium with the initial conditions in the form of a Poisson distribution of gravitating point masses demonstrate 
rather fast granulation (formation of clumped structure) with the sizes of both the granules (clumps) and the corresponding voids spanning the entire range of scale lengths from very short to extremely long. It is also known that the gravity force acting on a test particle and produced by a particular distribution of masses is determined both by the immediate environment of the particle and large-scale properties of the entire system. We already mentioned above that the classical Poisson model of the space distribution of masses implies the Holtsmark distribution for the magnitude of the random force acting on a test particle.

Unlike the uniform medium model used by Chandrasekhar (1943), the fractal gravitating medium of Carpenter-de Vaucouleurs-Mandelbrot contains multiscale correlation structures. These structures are characterized by stochastic self similarity and power-law conditional density distribution of the form of equation (1) inside them (Mandelbrot, 1977). This circumstance, in principle, makes it extremely difficult to analytically derive the random force distribution law in the general form. However, Vlad (1994) used a specially developed functional-and-integral approach to analyze non-uniform Poisson statistics, and applied his results to fractal structures generated by gravitational field. He analytically derived the distribution of random gravitational force without considering all real space density fluctuations. When computing the field-star density variations as a function of distance $r$ from the test particle, Vlad used (like we did in our case) the average densities in spheres of radius $r$ ignoring morphological peculiarities of particular structures (deviations from the mean distribution) which are usually present on any scale length. This approach is justified when studying a fractal structure and it allows such structures to be described in terms of correlation functions (Blumenfeld, Boll, 1993). In this approximation Vlad (1994) used n-point correlation functions to perform complex analytical manipulations to generalize the Holtsmark distribution and derive the following exact formula for the probability density $W(F)$ of random force magnitude:

$$
W(F) d F=\frac{D B}{2}(G m)^{\frac{D}{2}} F^{-\frac{D+2}{2}} \exp \left[-B(G m)^{\frac{D}{2}} F^{-\frac{D}{2}}\right] d F
$$

Here $D$ is the fractal dimension of the medium; , a constant that characterizes the average mass in a unit sphere, and $m$, the average mass of gravitating bodies.

Like in the case of our formula (14), uniform distribution corresponds to a fractal dimension of $D=3$. Thus for real distributions of gravitating points function $W(F)$ decreases slower with $\mathrm{F}$ than the corresponding function for uniform medium considered by Chandrasekhar (1947).

Formulas (14) and (21) fully coincide up to notation. This means that the distribution of random force in fractal gravitating media with a power-law conditional density variation is fully determined by the modified distribution of the nearest neighbor distance.

\section{Acknowledgments}

The study of the kinetics of stellar systems was supported by the Russian Foundation for Basic Research (grant no. 14-02-00472), and theoretical computations were supported by the Russian Science Foundation (grant no. 14-22-00041).

\section{REFERENCES}

Allen, C.W. Cox, A.N. 2004, Allen's Astrophysical Quantities, New York: IAP Press: Springer 
Bahcall, J. Soneira, R. 1986, Ann. Rev. Astron. Astrophys., 24, 577

Blumenfeld, R. Ball,R.C. 1993, Phys. Rev E. 47, 2298

Bottaccio,M. Montuori,M. Pietronero,L. Miocchi P.and Capuzzo Dolcetta,R. 2003, Mem. S.A.It. Suppl., 1, 120

Vlad, M.O. 1994, Astrophys. Space Sci., 218, 159

de Vaucouleurs, G. 1970, Science, 167, Is. 3922, 1203

de Vaucouleurs, G. et al., 1991, Third Reference Catalogue of Bright Galaxies. Volume I: Explanations and references. Volume II: Data for galaxies between Oh and 12h. Volume III: Data for galaxies between 12h and 24h Springer

Wu, K.S. Lahav, O. Rees, M. 1999, Nature, 397, Is. 6716, 225

Gouliermis, D.A.Hony, S. Klessen, R.S. 2014, MNRAS, 439, 3775

Gurevich, L.E. 1954, Evolyutsiya zvesdnykh system (Evolution of stellar systems). - In: Problems of cosmogono. V.2, Moscow (in Russian)

de Vega, H. Sanchez, N. Combes, F. Astrophys. J. 1998, 500, 8

Efremov, Yu.N. Elmegreen, B.G. 1998, MNRAS 299, 588

Carpenter, E.F. 1938, Astrophys. J., 88, 344

Larson, R.B. 1981, MNRAS, 194, 809

Mandelbrot, B.B. 1977, Fractals: Form, Chance and Dimension, W.H. Freedman\&Co., San Francisco.

Nordström, B. Mayor, M. Andersen 2004, J. J., et al. , A\&A, 418, 989

Ogorodnikov, K.F. 1958, Dynamic of the Stellar Systems Moskow (in Russian)

Robin, A. Reyle, C. Derriere S. 2003, Picaud S., A\&A, 409, 523

Sánchez, N. A?ez, N. Alfaro E.J. and Crone Odekon, M. strophys. J. 2010, 720, 541

Spitzer, L. Shvarzhild, M. 1953, Astrophys. J., 118, 1, 106

Semelin, B. Combes, F. 2000, A\&A, 360,1096

Semelin, B. Combes, F. 2002, A\&A, 387, 98

Holmberg, J. Nordström, B. Andersen, J. 2007, A\&A, 475, 519 )

Holmberg, J. Nordström, B. Andersen, J. 2009, A\&A, 501, 941

Chandrasekhar, S. 1943, Rev. Mod. Phys., 15, 1

Chandrasekhar, S. 1943, Stochastic Problems in Physics and Astronomy, Reviews of Modern Physics, 15, 1-89

Chumak, O.V. Rastorguev, A.S. 2015, Baltic Astron., 24, 1, 302

Chumak, O.V. Chumak, Z.N. 1976, Tr. Astrofiz. Inst., Akad. Nauk Kaz. SSR, 28, 81 (in Russian)

Chumak, O.V. Rastorguev, A.S. 2014, Pis'ma Astron. Zh., 40, 8, 517

Elias, F. Alfaro, E.J. Cabrero-Caño, J. 2009, MNRAS, 397, 2

Elmegreen,D.B. Elmegreen, B.G.Adamo A. et al., 2014, Astrophys. J. Letters, 787, L.15

Yanny, B. Gardner, S. 2013, Astrophys.J., 777, art. id.91 